\title{
Race modifies default mode network connectivity in Alzheimer's disease
}

Misiura, Maria B. ${ }^{1,2}$, Wu, Junjie ${ }^{3}$, Qiu, Deqiang ${ }^{3}$, Howell, Jennifer C. ${ }^{2}$, Parker, Monica W., ${ }^{2}$

Turner, Jessica A. ${ }^{1}, \mathrm{Hu}$, William T. ${ }^{2 *}$

${ }^{1}$ Department of Psychology, Georgia State University. Atlanta, GA

Departments of ${ }^{2}$ Neurology and ${ }^{3}$ Radiology, Emory University. Atlanta, GA

Manuscript classification: Article (Smaller Scope)

Title character count: 70

Running title character count: 41

Word count (not including Abstract): 1179

Introduction word count: 212

Abstract word count: 203

Total figure: 1

Table: 2

References: 10

Supplemental information: e-Methods, Table e-1

Keywords: [25] all cognitive disorders/dementia; [26] Alzheimer's disease; [120] MRI; [121] fMRI.

Statistical analysis: MBM and WTH are responsible for the statistical analysis.

Study funding: This study was supported by National Institutes of Health R21AG043885, R01AG054046, and P50AG025688.

Running title: Race modifies AD-related DMN connectivity

\author{
*Corresponding author: \\ William $\mathrm{Hu}, \mathrm{MD}, \mathrm{PhD}$ \\ Department of Neurology, Emory University \\ 615 Michael Street, 505F \\ Atlanta, GA 30322 \\ Phone 404-727-4174 \\ Email: wthu@emory.edu
}




\section{Funding}

This work was supported by National Institutes of Health grants AG43885, AG42856, and AG25688.

\section{Author Disclosures}

Ms. Misiura has nothing to disclose.

Dr. Wu has nothing to disclose.

Dr. Qiu has received research support from

Ms. Howell has nothing to disclose.

Dr. Parker has nothing to disclose.

Dr. Turner has nothing to disclose.

Dr. Hu has received grant support from Fujirebio US and Avid Radiopharmaceutical; has received travel support from Hoffman-LaRoche and Abbvie; has a patent on CSF-based diagnosis of neurodegenerative disorders (assigned to Emory University); consults for AARP Inc., ViveBio, LLC, Locks Law Firm, and Interpleader Law. 


\begin{abstract}
Objective: To determine if resting state functional MRI biomarkers of Alzheimer's disease (AD) differ between older African Americans and Caucasians.
\end{abstract}

Methods: We analyzed MRI profiles from 78 individuals (31 African Americans, 47

Caucasians) with normal cognition $(n=36)$ or mild cognitive impairment/mild AD dementia (MCI/AD, $\mathrm{n}=42$ ). We compared AD-associated intra-network functional connectivity of the default mode network (DMN) according to race, and correlated domain-specific cognitive functions with functional connectivity which differed between the racial groups.

Results: We identified key differences in DMN functional connectivity associated with AD between the races. Whereas MCI/AD was associated with decreased functional connectivity within the midline core DMN subsystem in older Caucasians, MCI/AD was instead associated with increased functional connectivity within the same subsystem of older African Americans. This is despite decreased functional connectivity in the medial temporal lobe DMN subsystem in both races. Memory function was also positively associated with connectivity between the precuneus and the posterior cingulate/inferior parietal lobule within the midline core subsystem, in keeping with a less amnestic-profile in older African Americans with MCI/AD.

Conclusions: These findings provide structural support that race modifies the AD phenotypes downstream from cerebral amyloid deposition, and suggests a rsf-MRI correlate of African American's less amnestic neuropsychological profile in MCI/AD. 


\section{Introduction}

Older African Americans are twice as likely to develop Alzheimer's disease (AD) as older non-Hispanic Caucasians. ${ }^{1}$ Epidemiological and genome-wide association studies in clinically defined cases of dementia suggest that AD may manifest differently according to race, vascular risks, educational duration, and susceptibility genes. ${ }^{1,2}$ We previously reported that AD was associated with lower CSF tau-related biomarker levels in African Americans than Caucasians, despite similar levels of beta-amyloid 1-42 (A $\beta 42) .{ }^{2}$ We did not find a difference in hippocampal volumes, but it is not known if other objective biomarkers differed between the two races. Here we analyze MRI biomarkers that may reflect earlier AD-related processes and may better inform the relationship between race and AD neuroimaging phenotypes.

In $\mathrm{AD}$, disrupted functional connectivity in the default mode network (DMN) is reported to occur following amyloid biomarker changes but before significant hippocampal atrophy. ${ }^{3,4}$ We hypothesized that, similar to baseline and AD-associated differences in CSF tau biomarkers between the two racial groups, older African Americans also had AD-associated DMN alterations distinct from older Caucasians. To test this hypothesis, we analyzed resting state functional (rsf-) MRI from the same group of subjects with normal cognition (NC) or early AD (mild cognitive impairment or mild AD dementia, $\mathrm{MCI} / \mathrm{AD}$ ), and analyzed if race, diagnosis, and other factors influenced DMN connectivity measures.

\section{Methods}

Standard Protocol Approvals, Registrations, and Patient Consents: This study was approved by the Emory University Institutional Review Board and written consent was obtained from all subjects. 
Participants: We included 78 participants who underwent uniform clinical, neuropsychological, genetic, and CSF biomarker analysis as previously described (Table e-1). ${ }^{2}$ MRI analysis was performed using a modified Alzheimer's Disease Neuroimaging Initiative (ADNI) protocol (eMethods). For functional connectivity, regions of the DMN include the precuneus (PCUN), posterior cingulate gyrus/inferior parietal lobule (PCC/IPL), the ventromedial prefrontal cortex (vmPFC), and the hippocampus. We used a data driven approach (Independent Component Analysis; ICA) using the Group ICA of fMRI Toolbox (GIFT) to identify large-scale brain networks, and calculated connectivity measures between DMN nodes. ${ }^{5}$

\section{Data availability}

The data that support the findings of this study are available from the corresponding author, upon reasonable request.

\section{Statistical Analyses}

Statistical analysis was performed in $\mathrm{R}$ version 3.3.3. We constructed multivariate multiple linear regression models with intra-network connectivity between the three posterior DMN nodes as the dependent variables; race, sex, and diagnosis (normal cognition, MCI/AD), age, and mean framewise displacement (MFWD) as independent variables, as well as a higher order interaction term (race X diagnosis). Measures of connectivity between the anterior DMN component (vmPFC) and the hippocampus, PCUN, and PCC/IPL were similarly analyzed for raceassociated differences. We additionally examined the relationship of potential confounding effects of $A P O E \varepsilon 4, A B C A 7$ risk allele, hypertension, and diabetes which differed between the races. $^{2}$ 
To determine possible functional consequences of race-associated differences in DMN connectivity, we also correlated DMN connectivity that differed between the races with domainspecific cognitive functions. In this model, domain-specific Z-scores for executive, memory, language, and visual spatial function were the dependent variable, and diagnosis (NC vs. $\mathrm{MCI} / \mathrm{AD})$, connectivity, age, and having at least one $A P O E \varepsilon 4$ allele were the independent variables.

\section{Results}

\section{Race modifies the relationship between MCI/AD and midline core DMN connectivity}

Consistent with published results, $\mathrm{MCI} / \mathrm{AD}$ was associated with reduced anterior-posterior DMN connectivity (vmPFC-PCC/IPL and vmPFC-PCUN) and unchanged PCUN-PCC/IPL connectivity in older Caucasians, adjusting for age and gender (Fig 1A). Race modified the relationship between $\mathrm{MCI} / \mathrm{AD}$ diagnosis and connectivity for all three examined midline node pairs. Unlike in Caucasians, MCI/AD was associated with increased PCUN-PCC/IPL connectivity in African Americans, and unchanged anterior-posterior DMN connectivity. Further adjustment for $A B C A 7$ and $A P O E$ genotypes showed similar results (Table 1).

\section{Race does not modify the relationship between MCI/AD and medial temporal DMN connectivity}

Also consistent with published results, MCI/AD was associated with reduced connectivity involving the hippocampus in Caucasians (adjusting for age and gender, Fig 1B; hippocampus-PCUN, $F(2,75)=2.13, p=0.05$; hippocampus-PCC/IPL, $F(2,75)=2.17, p=0.04)$. Race did not influence hippocampal DMN connectivity, nor did it modify the relationship between $\mathrm{MCI} / \mathrm{AD}$ and hippocampal connectivity. 


\section{Greater vmPFC-PCUN connectivity is associated with better memory}

Finally, we determined if the connectivity profile differences between African Americans and Caucasians were associated with distinct neuropsychological profiles. Across the entire cohort, greater PCUN-PCC/IPL connectivity correlated with better memory functions independent of race (Table 2). Because increased PCUN-PCC/IPL connectivity was associated with MCI/AD only in African Americans, these results together are in keeping with less amnestic MCI/AD profiles (at the group level) in African Americans than Caucasians.

\section{Discussion}

The biological underpinning for epidemiologic and phenotypic differences in AD between older African Americans and Caucasians remains poorly defined. Here we show that race altered MCI/AD-associated connectivity profiles in the midline core DMN subsystem but not the amyloid-associated medial temporal subsystem. These differences cannot be readily explained by genetic risks from $A B C A 7$ and $A P O E$ alone, but likely have clinically meaningful impact as increased posterior midline DMN connectivity in African Americans with MCI/AD corresponds to their less amnestic neuropsychological profiles.

Race-related AD phenotypic differences have historically been attributed to education quality, access to health care, and cerebrovascular disease. ${ }^{6}$ The lower CSF tau levels in older African Americans regardless of cognitive status cannot be adequately explained by these explanations, ${ }^{2}$ and may correlate with midline DMN connectivity profiles. It remains controversial whether altered midline core connectivity reflects post mortem or in vivo measures of tau pathology, ${ }^{8}$ but this subsystem correlates only marginally with cerebral amyloid deposition. ${ }^{7}$ Increased DMN 
connectivity was previously linked to non-AD neurodegenerative diseases, and it is possible that greater non-AD co-pathology in African American $\mathrm{AD}$ cases on autopsy begins early rather than late following cerebral amyloid deposition. ${ }^{9}$ Alternatively, increased connectivity may represent network compensation, which suggests greater - not less - cognitive reserve in African Americans with $\mathrm{AD}$ to account for their slower clinical progression. ${ }^{10}$ Because there is significant overlap between the groupings, future work should prospectively analyze the relationship between tau PET tracer retention, CSF tau levels, and DMN connectivity in both Caucasians and African Americans. Because African Americans with MCI/AD also have a slower cognitive decline than Caucasians, PCUN-PCC/IPL connectivity - increased at the group level in African Americans - should also be tested as a prognostic biomarker in longitudinal studies.

While these findings extend our previous description of a specific AD endophenotype in African Americans, this study was limited by the number of rsf-MRI scans of insufficient quality, the association nature of the relationships between race and connectivity, and lack of neuropathologic correlate for connectivity differences. Nevertheless, when interpreted with previous data, this study lends further support to the notion that amyloid-related changes -CSF A $\beta 42$ level, and medial temporal DMN connectivity - are common to African Americans and Caucasians in AD, but tau-related changes - CSF tau levels, midline core DMN connectivity, and clinical progression - are modified by race. Not only do these findings promote caution when generalizing AD diagnostic biomarkers developed in Caucasians to African Americans, they may also help refine the subject selection process for future disease modifying therapies. 


\section{Acknowledgements}

The authors acknowledge the following persons for assistance with enrollment: James Lah, MD,

PhD; Allan Levey, MD, PhD; Chadwick Hales, MD, PhD; Angela Ashley, MD; Felicia

Goldstein, $\mathrm{PhD}$; and Andrea Kippels, CNP. 


\section{Appendix 1. Author Contributions}

\begin{tabular}{|c|c|c|c|}
\hline Name & Location & Role & Contribution \\
\hline $\begin{array}{l}\text { Maria B. Misiura, } \\
\text { M.A. }\end{array}$ & $\begin{array}{l}\text { Psychology } \\
\text { Department, Georgia } \\
\text { State University. } \\
\text { Atlanta, GA }\end{array}$ & Author & $\begin{array}{l}\text { Designed and } \\
\text { conceptualized study; } \\
\text { analyzed the data; } \\
\text { drafted the manuscript } \\
\text { for intellectual content }\end{array}$ \\
\hline Junjie $\mathrm{Wu}, \mathrm{PhD}$ & $\begin{array}{l}\text { Department of } \\
\text { Radiology, Emory } \\
\text { University. Atlanta, } \\
\text { GA }\end{array}$ & Author & $\begin{array}{l}\text { Major role in data } \\
\text { acquisition and } \\
\text { analysis }\end{array}$ \\
\hline Deqiang Qiu & $\begin{array}{l}\text { Department of } \\
\text { Radiology, Emory } \\
\text { University. Atlanta, } \\
\text { GA }\end{array}$ & Author & $\begin{array}{l}\text { Designed the study; } \\
\text { major role in imaging } \\
\text { data acquisition and } \\
\text { analysis }\end{array}$ \\
\hline Jennifer Howell & $\begin{array}{l}\text { Department of } \\
\text { Neurology, Emory } \\
\text { University. Atlanta, } \\
\text { GA }\end{array}$ & Author & $\begin{array}{l}\text { Major role in data } \\
\text { acquisition and } \\
\text { analysis }\end{array}$ \\
\hline $\begin{array}{l}\text { Monica W. Parker, } \\
\text { MD }\end{array}$ & $\begin{array}{l}\text { Department of } \\
\text { Neurology, Emory } \\
\text { University. Atlanta, } \\
\text { GA }\end{array}$ & Author & $\begin{array}{l}\text { Major role in data } \\
\text { acquisition }\end{array}$ \\
\hline Jessica A Turner, PhD & $\begin{array}{l}\text { Psychology } \\
\text { Department, Georgia } \\
\text { State University. } \\
\text { Atlanta, GA }\end{array}$ & Author & $\begin{array}{l}\text { Assisted in the } \\
\text { conceptualization of } \\
\text { the study; edited the } \\
\text { manuscript for critical } \\
\text { intellectual content }\end{array}$ \\
\hline $\begin{array}{l}\text { William Hu, MD, } \\
\text { PhD }\end{array}$ & $\begin{array}{l}\text { Department of } \\
\text { Neurology, Emory } \\
\text { University. Atlanta, } \\
\text { GA }\end{array}$ & Author & $\begin{array}{l}\text { Major role data } \\
\text { acquisition; assisted } \\
\text { in the } \\
\text { conceptualization of } \\
\text { the study; analyzed } \\
\text { the data; edited the } \\
\text { manuscript for critical } \\
\text { intellectual content }\end{array}$ \\
\hline
\end{tabular}




\section{References}

1. Barnes, L. L. \& Bennett, D. A. Alzheimer's Disease In African Americans: Risk Factors And Challenges For The Future. Health Aff. Proj. Hope 33, 580-586 (2014).

2. Howell, J. C. et al. Race modifies the relationship between cognition and Alzheimer's disease cerebrospinal fluid biomarkers. Alzheimers Res. Ther. 9, 88 (2017).

3. Hafkemeijer, A., van der Grond, J. \& Rombouts, S. A. R. B. Imaging the default mode network in aging and dementia. Imaging Brain Aging Neurodegener. Dis. 1822, 431-441 (2012).

4. Damoiseaux, J. S. Resting-state fMRI as a biomarker for Alzheimer's disease? Alzheimers Res. Ther. 4, 8-8 (2012).

5. Calhoun, V. D. \& Adali, T. Multisubject Independent Component Analysis of fMRI: A Decade of Intrinsic Networks, Default Mode, and Neurodiagnostic Discovery. 5, (2012).

6. Miles, T. P., Froehlich, T. E., Bogardus, S. T. \& Inouye, S. K. Dementia and Race: Are There Differences Between African Americans and Caucasians? J. Am. Geriatr. Soc. 49, 477-484 (2001).

7. Jones, D. T. et al. Cascading network failure across the Alzheimer's disease spectrum. Brain 139, 547-562 (2016).

8. Hansson, O. et al. Tau Pathology Distribution in Alzheimer's disease Corresponds Differentially to Cognition-Relevant Functional Brain Networks. Front. Neurosci. 11, 167 (2017).

9. Zhou, J. et al. Divergent network connectivity changes in behavioural variant frontotemporal dementia and Alzheimer's disease. Brain 133, 1352-1367 (2010). 
bioRxiv preprint doi: https://doi.org/10.1101/472910; this version posted November 19, 2018. The copyright holder for this preprint (which was not certified by peer review) is the author/funder. All rights reserved. No reuse allowed without permission.

10. Simioni, A. C., Dagher, A. \& Fellows, L. K. Compensatory striatal-cerebellar connectivity in mild-moderate Parkinson's disease. NeuroImage Clin. 10, 54-62 (2016). 
Table 1. Effects of race, MCI/AD, and risk genes/factors on DMN connectivity. Note: N.S. = not significant

\begin{tabular}{|l|c|c|c|}
\hline & vmPFC-PCUN & vmPFC-PCC/IPL & PCUN-PCC/IPL \\
& $(\mathrm{F}, \mathrm{p})$ & $(\mathrm{F}, \mathrm{p})$ & $13.700(<0.001)$ \\
\hline Race $x \mathrm{MCI} / \mathrm{AD}$ & $3.150(0.02)$ & $3.091(0.01)$ & N.S. \\
\hline APOE $\varepsilon 4$ & $4.079(0.047)$ & $3.499(0.065)$ & $5.328(0.024)$ \\
\hline ABCA7 & $3.511(0.065)$ & N.S. & N.S. \\
\hline Hypertension & $3.063(0.084)$ & N.S. & N.S. \\
\hline Diabetes & N.S. & N.S. & 3.009 (0.056) \\
\hline Diabetes x MCI/AD & N.S. & $2.497(0.067)$ & \\
\hline
\end{tabular}


Table 2. Effects of diagnosis, risk genes/factors, and DMN connectivity on memory.

\begin{tabular}{|l|l|l|}
\hline Variable & $\begin{array}{l}\text { Coefficient }(95 \% \\
\text { confidence interval) }\end{array}$ & P \\
\hline MCI/AD diagnosis & $-1.362(-1.942,-0.782)$ & $<0.001$ \\
\hline Having at least one APOE $\varepsilon 4$ allele & $-0.861(-1.441,-0.280)$ & 0.004 \\
\hline Age & $0.066(0.026,0.105)$ & 0.001 \\
\hline PCUN-PCC/IPL connectivity & $1.235(0.124,2.347)$ & 0.030 \\
\hline
\end{tabular}




\section{Figure legend}

Figure 1.Connectivity and diagnosis. Y-axes for all graphs are functional connectivity. Bars represent medians for each group. Panel A depicts connectivity and diagnosis relationships among the PCUN, PCC/IPL, and vmPFC nodes. MCI/AD was associated with reduced anteriorposterior DMN connectivity (vmPFC-PCC/IPL and vmPFC- PCUN) in Caucasians but not African Americans. In contrast, MCI/AD was associated with increased PCUN-PCC/IPL connectivity in African Americans but not Caucasians. Panel B depicts connectivity between the hippocampi and midline core DMN nodes. Unlike in A, MCI/AD was associated with reduced connectivity between the hippocampus and midline core nodes irrespective of race. 
A

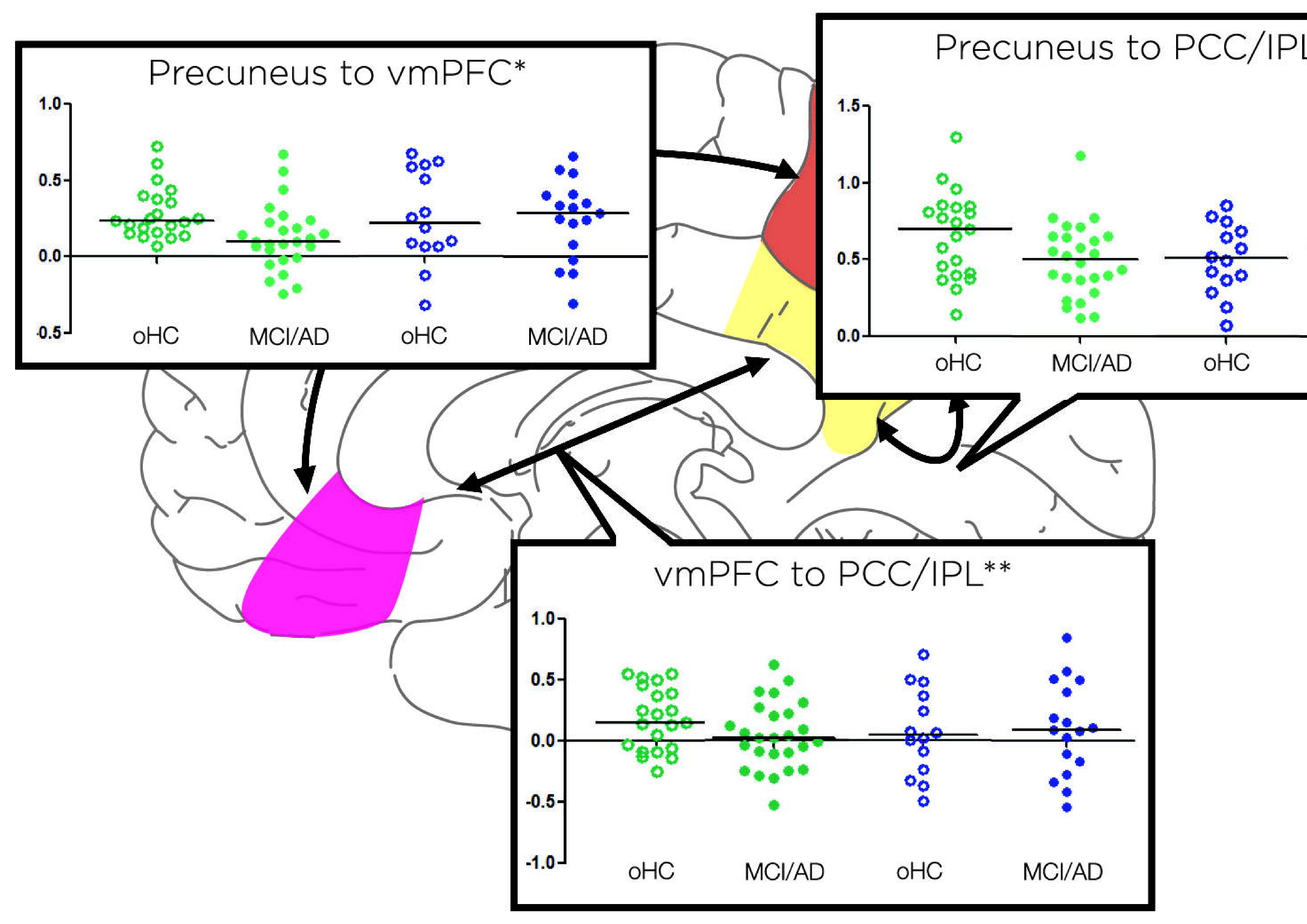

\section{Legend}

Precuneus

Hippocampus PCC/IPL vmPFC

${ }^{*} \mathrm{p}<.05$ for Race $X$ Diagnosis interaction ** $p<01$ for Race X Diagnosis interaction

Non-Hispanic White: Green

- Older Healthy Control

- $\mathrm{MCl} / \mathrm{AD}$

B

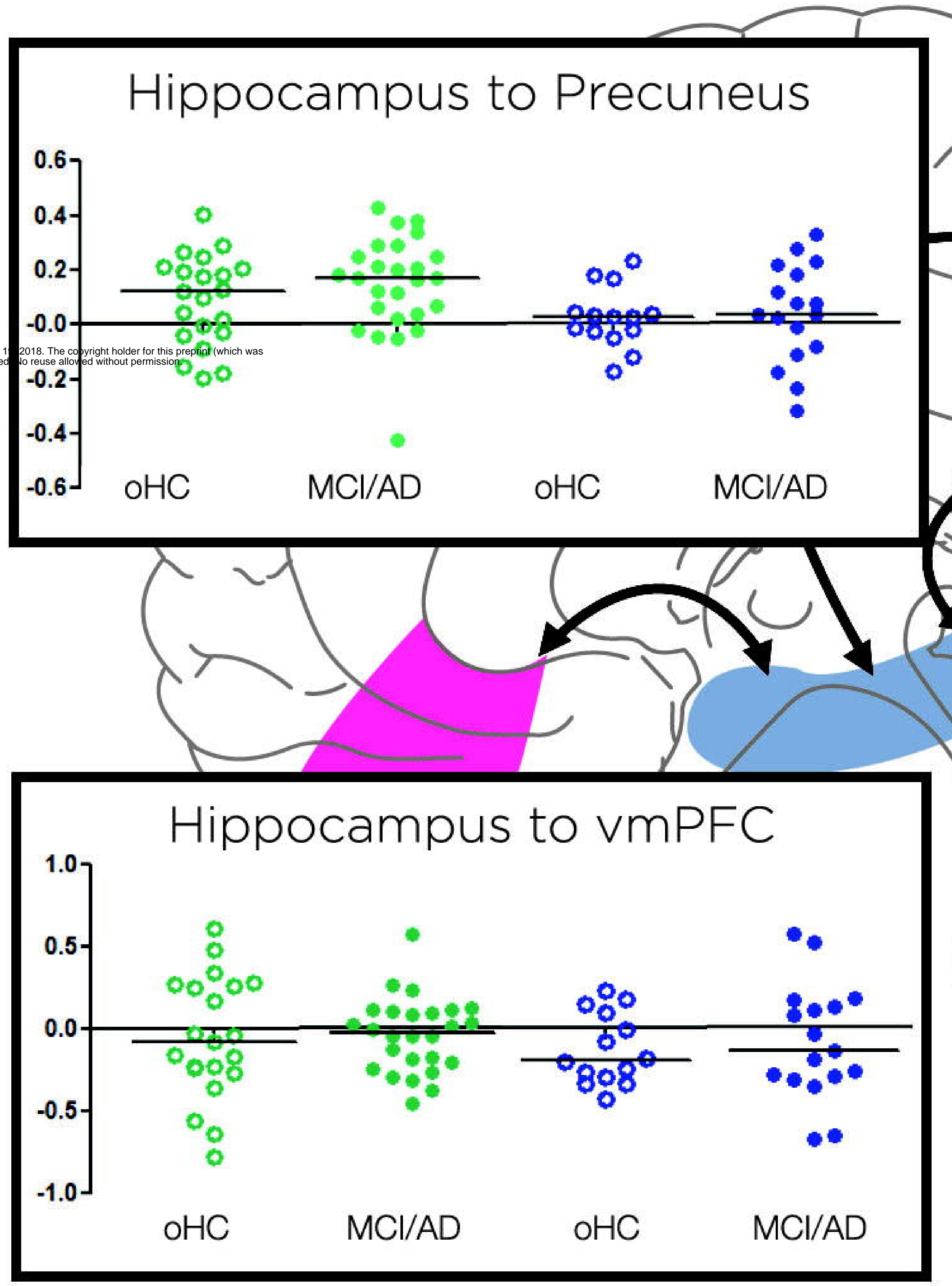

African American: Blue

- Older Healthy Control

- $\mathrm{MCl} / \mathrm{AD}$ 